\title{
Scanning electron microscopy of polycystic liver disease
}

\author{
Kazutaka Kojima $^{1,2} \cdot$ Masaji Hashimoto $^{1} \cdot$ Yoshifumi Ubara $^{2,3}$
}

Received: 14 November 2017 / Accepted: 20 February 2018 / Published online: 8 March 2018

(c) The Author(s) 2018. This article is an open access publication

\begin{abstract}
Resected specimens of PCLD by laparoscopic fenestration surgery were evaluated by scanning electron microscopy. Epithelium lining the largest cyst ( $26 \mathrm{~cm}$ in size) showed prominent villous proliferation with positivity of Ki-67, while the epithelium of the small cyst ( $3 \mathrm{~cm}$ in size) showed slight proliferation (smooth) with small positivity of Ki-67.
\end{abstract}

Keywords Polycystic liver disease (PCLD) - ADPKD (autosomal dominant polycystic kidney disease) · Fenestration surgery $\cdot$ Scanning electron microscopy

Fig. 1 Computed tomography showed a huge cyst, and contracted kidneys with multiple small cysts. Laparoscopic fenestration surgery was performed for huge cyst measuring $26.1 \mathrm{~cm} \times 23.7 \mathrm{~cm} \times 16.1 \mathrm{~cm}$ (large arrow) and small cyst measuring $3.1 \mathrm{~cm} \times 2.9 \mathrm{~cm} \times 2.6 \mathrm{~cm}$ (small arrow). a Axial section, b coronal section

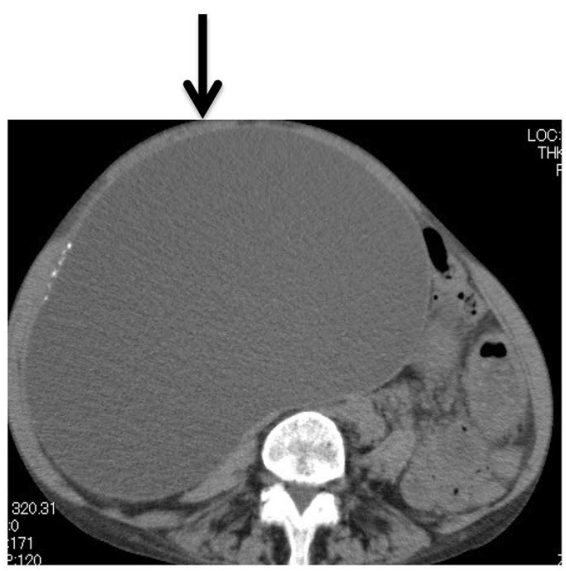

(a)

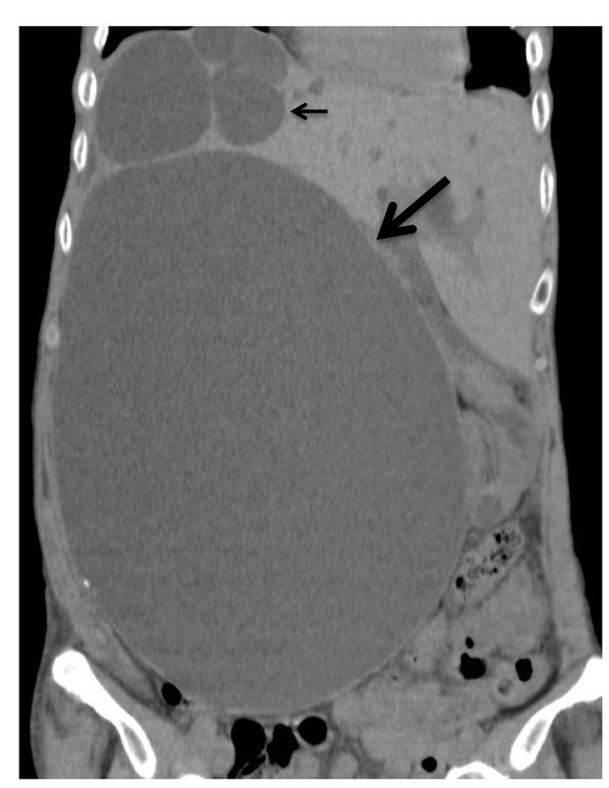

(b)

Yoshifumi Ubara

ubara@toranomon.gr.jp

1 Department of Gastrointestinal Surgery, Toranomon Hospital, Tokyo, Japan

2 Nephrology Center, Toranomon Hospital Kajigaya, 1-3-1, Kajigaya, Takatsu, Kawasaki 213-8587, Kanagawa, Japan

3 Okinaka Memorial Institute for Medical Research, Toranomon Hospital, Tokyo, Japan 

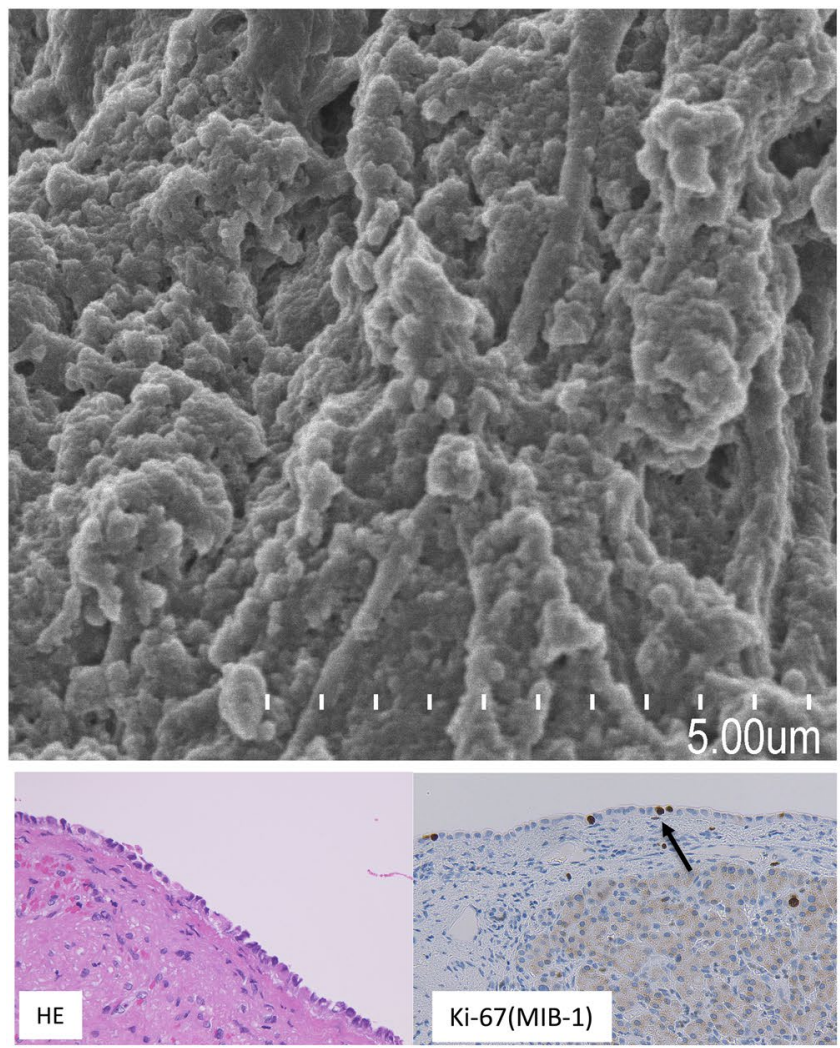

(a)

Fig. 2 Scanning electron microscopy of the largest cyst a and small cyst, b shows prominent villous proliferation (without cilium) (a) and slight proliferation (with cilium) (large arrow) (b), respectively. Inset shows hematoxylin and eosin stain (HE) and Ki-67 (MIB-1) of cyst

A 69-year-old woman with ADPKD was admitted to our hospital for evaluation of abdominal distention due to PCLD. Resected specimens by laparoscopic fenestration surgery were evaluated by scanning electron microscopy. Epithelium lining the largest cyst (26 cm in size) (Fig. 1a) showed prominent villous proliferation with positivity of Ki-67 (Fig. 2a), while the epithelium of the small cyst $(3 \mathrm{~cm}$ in size) (Fig. 1b) showed slight proliferation (smooth) with small positivity of Ki-67 (Fig. 2b). We believe that villous change of the cyst epithelium may be closely associated with largest cyst volume via the proliferation of cyst lining cells in PCLD.

Acknowledgements We thank Drs. Hiroki Mizuno, Masahiko Oguro, Yoichi Oshima, Tatsuya Suwabe, Keiichi Sumida, Masahiro Kawada, Masayuki Yamanouchi, Akinari Sekine, Rikako Hiramatsu,Eiko Hasegawa, Noriko Hayami, Junichi Hoshino, Naoki Sawa and Kenmei Takaichi (Nephrology Center, Toranomon Hospital), Keiichi Kinowaki, Takeshi Fujii (Pathology department, Toranomon Hospital)and Kanae Yamamoto (Department of Nephrology, Graduate School of Medicine, Kyoto University).
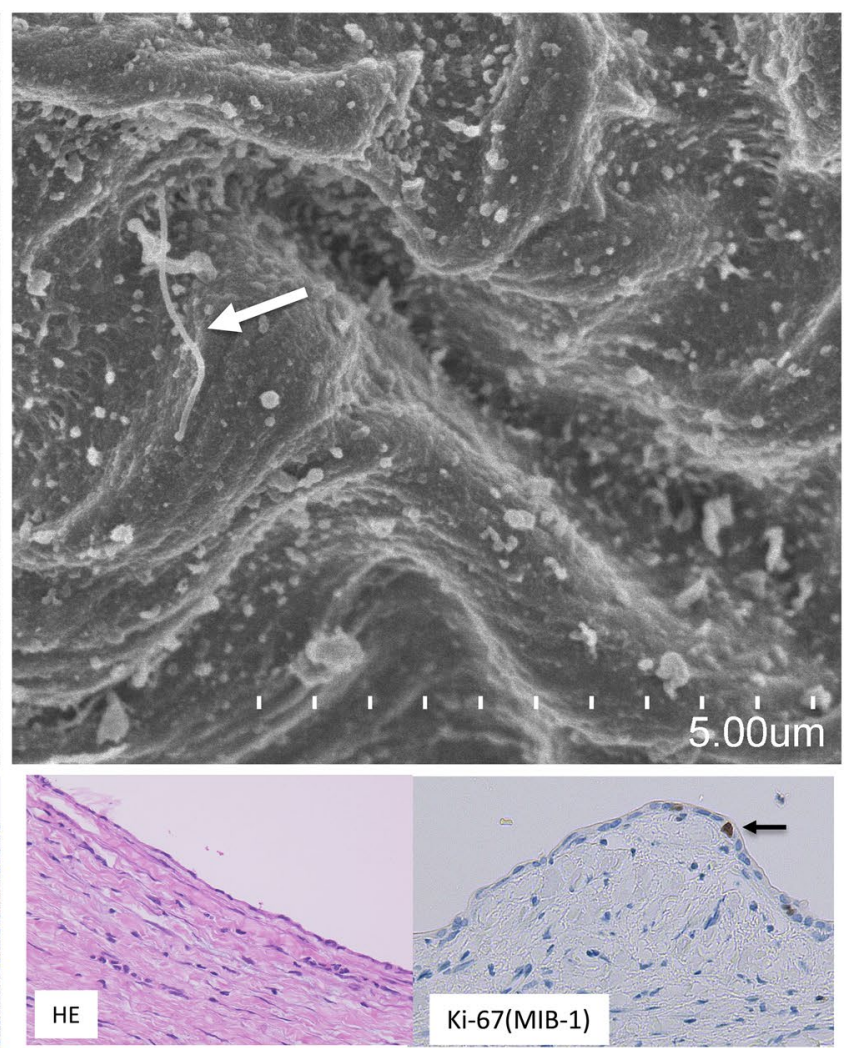

(b)

wall; a the largest cyst shows high length epithelium with positivity of Ki-67 (small arrows), and b small cyst shows flat epithelium with small positivity of Ki-67 (small arrow)

\section{Compliance with ethical standards}

Conflict of interest The authors report no conflicts of interest.

Ethical approval All procedures performed in studies involving human participants were in accordance with the ethical standards of the institutional and/or national research committee at which the studies were conducted (IRB approval number 1379-B) and with the 1964 Helsinki declaration and its later amendments or comparable ethical standards.

Informed consent Informed consent was obtained from individual participant included in this study.

Open Access This article is distributed under the terms of the Creative Commons Attribution 4.0 International License (http://creativeco mmons.org/licenses/by/4.0/), which permits unrestricted use, distribution, and reproduction in any medium, provided you give appropriate credit to the original author(s) and the source, provide a link to the Creative Commons license, and indicate if changes were made. 